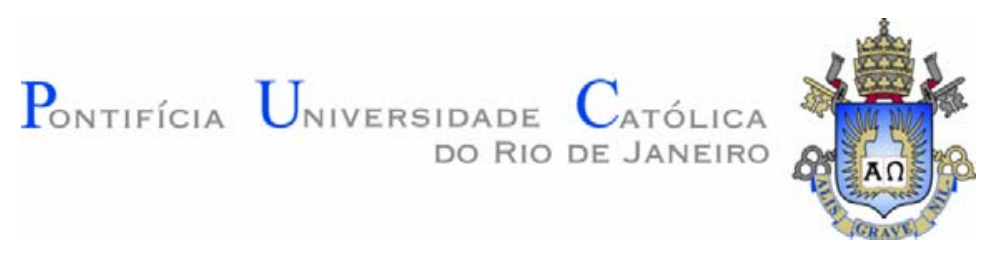

Marlene Susy Tapia Morales

\title{
Análise de confiabilidade de taludes em condições saturadas- não saturadas via análise limite no espaço cônico quadrático
}

Tese de Doutorado

Tese apresentada como requisito parcial para obtenção do titulo de Doutor pelo Programa de PósGraduação em Engenharia Civil do Departamento de Engenharia Civil da PUC-Rio.

Orientador: Prof. Eurípedes do Amaral Vargas Júnior Co-orientador: Prof. Luiz Eloy Vaz

Rio de Janeiro

Setembro de 2013 


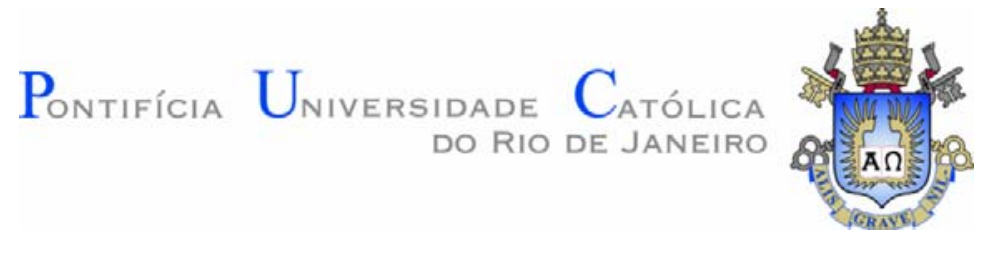

Marlene Susy Tapia Morales

\section{Análise de confiabilidade de taludes em condições saturadas- não saturadas via análise limite no espaço cônico quadrático}

Tese apresentada como requisito parcial para obtenção do grau de Doutor pelo Programa de Pós-graduação em Engenharia Civil do Departamento de Engenharia Civil da PUCRio. Aprovada pela Comissão Examinadora abaixo assinada.

Prof. Eurípedes do Amaral Vargas Júnior Orientador Departamento de Engenharia Civil - PUC-Rio

Prof. Luiz Eloy Vaz Co-Orientador Universidade Federal Fluminense

Prof. Gilson de Farias Neves Gitirana Júnior Universidade Federal de Goiás

Prof. Tacio Mauro Pereira de Campos Departamento de Engenharia Civil - PUC-Rio

Doutor Raquel Quadros Velloso Departamento de Engenharia Civil - PUC-Rio

Doutor. Andre Maues Brabo Pereira Universidade Federal Fluminense

Prof. José Eugenio Leal Coordenador Setorial do Centro Técnico Cientifico - PUC-Rio 
Todos os direitos reservados. É proibida a reprodução total ou parcial do trabalho sem autorização da universidade, do autor e do orientador.

\section{Marlene Susy Tapia Morales}

Graduou-se em Engenharia Civil na Universidad Nacional San Antonio Abad del Cusco (Perú), possui mestrado em Engenharia Civil pela Pontifícia Universidade Católica de Rio de Janeiro (PUC-Rio), com ênfase em Geotecnia Ambiental.

Ficha Catalogrâfica

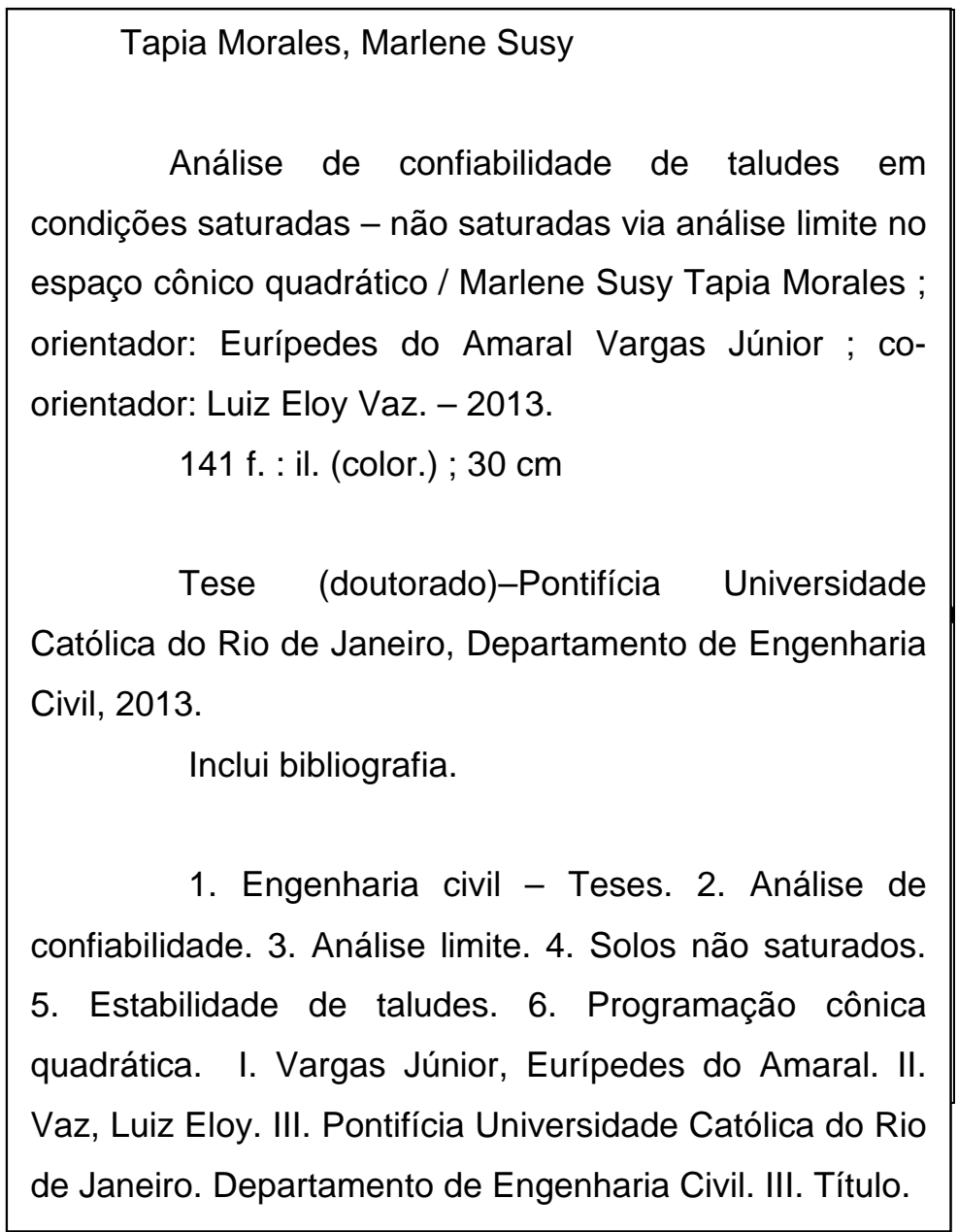

CDD: 624 
A meus queridos e amados pais, Javier e Sabina, minhas irmãs Rosa, Licelly e Thalia pelo que significam, tudo para mim... 


\section{Agradecimentos}

Ao $\mathrm{CNPq}$ e ao Departamento de Engenharia Civil da PUC-Rio, pela ajuda financeira e oportunidade concedida na minha evolução profissional, sem os quais este trabalho não poderia ter sido realizado.

À meu orientador Eurípedes do Amaral Vargas Júnior, pela oportunidade, orientação e conhecimentos transmitidos durante a elaboração deste trabalho.

À meu co-orientador Luiz Eloy Vaz pela oportunidade e a paciência em transmitir muito do seu valioso conhecimento durante o desenvolvimento de minha pesquisa.

À Raquel pelas inúmeras respostas que contribuíram para este trabalho.

À minha família pais e irmãs por todo o que passamos juntos para chegar até aqui, obrigado por ser meu porto seguro e a minha torcida oficial.

Ao Paul, por ser minha fonte de admiração, inspiração e por seu apoio incondicional em todos os momentos que passamos nesta árdua jornada; sem você isto não teria se tornado possível.

À Presvitero e Joana, pela sua amizade, apoio e incentivo para terminar este trabalho.

Aos meus amigos Liset, Liliana, Gerado, Jocileia e demais colegas pela amizade e pelos bons momentos compartilhados nestes anos.

Aos funcionários da Secretaria do DEC pela sempre amável atenção. 


\section{Resumo}

Tapia Morales, Marlene Susy; Eurípedes do Amaral Vargas Júnior; Luiz Eloy Vaz. Análise de confiabilidade de taludes em condições saturadasnão saturadas via análise limite no espaço cônico quadrático. Rio de Janeiro, 2013. 141p. Tese de Doutorado - Departamento de Engenharia Civil, Pontifícia Universidade Católica do Rio de Janeiro.

Este trabalho tem por objetivo a avaliação da estabilidade de taludes de solo quando sometidos a processos de infiltração de chuva, utilizando conceitos de Análise Limite e Análise de Confiabilidade. Primeiramente, determina-se a variação da sução no solo, para isto, emprega-se o Método dos Elementos Finitos e o Método de diferenças finitas na solução da equação de Richards. O modelo de Van Genuchten (1980) é utilizado para a curva característica. Na solução da nãolinearidade, emprega-se o método Picard Modificado. A instabilidade de taludes é estudada mediante o método de Análise Limite Numérica com base no Método de Elementos Finitos e o critério de Mohr Coulomb como critério de escoamento. A solução do problema matemático será realizada no espaço cônico quadrático com o objetivo de tornar a solução mais computacionalmente eficiente. Considerando as propriedades do solo como variáveis aleatórias foi incluída a determinação do Índice de Confiabilidade utilizando as formulações dos métodos de Monte Carlo e FORM (first order reliability method). Inicialmente são introduzidos conceitos básicos associados ao fluxo saturado-não saturado. A seguir são apresentados alguns conceitos. Sobre Análise Limite e sua formulação pelo Método de Elementos Finitos. Finalmente são introduzidos os fundamentos da Análise de Confiabilidade. Análises de confiabilidade das encostas de Coos Bay no estado de Oregon nos Estados Unidos e da Vista Chinesa no Rio de Janeiro Brasil, são apresentadas devido a que estes taludes sofreram colapso quando submetidos a processos de infiltração de água de chuva. Os resultados deste trabalho mostram que a falha das encostas ocorre quando o índice de confiabilidade atinge um valor perto de dois.

\section{Palavras-chave}

Análise Limite; Confiabilidade; Estabilidade de taludes; Programação cônica quadrática. 


\section{Abstract}

Tapia Morales, Marlene Susy; Eurípedes do Amaral Vargas Júnior (Advisor); Luiz Eloy Vaz (Co-Advisor). Reliability Analysis of saturatedunsaturated soil slopes using limit analysis in the conic quadratic space. Rio de Janeiro, 2013. 141p. Tese de Doutorado - Departamento de Engenharia Civil, Pontifícia Universidade Católica do Rio de Janeiro.

This thesis aims to perform a reliability analysis of the stability of 2D soil slopes when they are submitted to water infiltration due to the rains. The time variation of the soil matric suctions is calculated first. The Finite Element Method is used to transform the Richards differential equation into a system of nonlinear first order equations. The nonlinearity of the problem is due to the use of the characteristic curve proposed by van Genuchten (1980). The Modified Picard Method is applied to solve de time-dependent nonlinear equation system. The responses of the flux-problem are transferred to the stability problem in some instants using the same time-interval (normally days).To estimate the stability of the slopes, limit analysis is used. The limit analyses are performed based on the Inferior Limit Theorem of the Plasticity Theory. The problem is defined as an optimization problem where the load factor is maximized. The equilibrium equations are obtained via Finite Element discretization and the strength criterion of Mohr-Coulomb is written in the conic quadratic space. Therefore, a SOCP (Second Order Conic Programming) problem is generated. The problem is solved using an interior point algorithm of the code Mosek.Since the soil properties are random variables a reliability analysis can be performed at each instant of the time-dependent problem. In order to perform the reliability analyses, Response Surfaces for the failure function of the slope are generated. In this work, the Stochastic Collocation Method is used to generate Response Surfaces. The Simulation Monte Carlo Method and the FORM (First Order Reliability Method) are used to obtain both the reliability index and the probability of failure of the slopes.Reliability analyses of the Coos Bay Slope in the state of Oregon in USA and in the Vista Chinesa Slope in Rio de Janeiro, Brazil, are presented because they collapse due to rainfall infiltration. The results show that the soil slope fails when the related reliability index is close to two.

\section{Keywords}

Limit analysis; Reliability; Slope stability; Conic quadratic programming. 


\section{Sumário}

1 Introdução

2 Critérios de Resistência $\quad 22$

2.1. Modelos Constitutivos $\quad 22$

2.2. Critério de Resistência de Mohr-Coulomb 24

2.3. Criterio de Resistência de Drucker-Prager 26

2.4. Critério de Resistência em Solos não Saturados 27

3 Fluxo Saturado e não Saturado 31

3.1. Potencial da Água no Solo 32

3.2. Equação Governante 32

3.3. Propriedades Hidráulicas de Solos Não Saturados 36

3.3.1. Curva Característica 36

3.3.2. Curva de Condutividade Hidráulica 39

3.4. Solução Numérica da Equação de Fluxo 40

3.4.1. Discretização Espacial 43

3.4.2. Discretização no Tempo 45

3.4.3. Método Picard Modificado 46

3.4.4. Critério de Convergência 48

3.4.5. Exemplos de Validação 49

4 Análise Limite $\quad 54$

4.1. Teoremas de Análise Limite $\quad 55$

4.1.1. Teorema de Análise Limite Inferior 56

4.1.2. Teorema de Limite Superior 56

4.2. Conceitos Relacionados à Plasticidade 57

4.2.1. Critério de Resistência

4.2.2. Lei de Fluxo 58

4.2.3. Principio do Trabalho Virtual $\quad 59$

4.3. Formulação da Análise Limite pelo Método dos Elementos Finitos 59

4.4. Formulação da Equação de Equilíbrio 60

4.4.1. Formulação Forte 60 
4.4.2. Formulação Fraca 60

4.4.3. Condição de Equilíbrio e Compatibilidade 61

4.4.4. Condições de Contorno 63

4.4.5. Condição de Resistência 63

4.5. Formulação Convencional do Problema de Análise Limite Inferior 64

4.6. Formulação no Espaço Cônico Quadrático da Análise Limite 64

4.6.1. Problema Cônico Quadrático 65

4.6.2. Critério de Resistência de Mohr-Coulomb no Espaço Cônico Quadrático 66

4.7. Elemento Finito Implementado $\quad 67$

4.8. Exemplos de Validação $\quad 69$

4.8.1. Talude Infinito Homogêneo 69

4.8.2. Exemplo de Talude 2D 71

5 Análise de Confiabilidade $\quad 74$

5.1. Fundamentos $\quad 75$

5.2. Função de Falha $\quad 75$

5.2.1. Probabilidade de Falha 76

5.2.2. Índice de Confiabilidade 76

5.3. Métodos de Análise de Confiabilidade $\quad 80$

5.3.1. Método de Simulação de Monte Carlo 80

5.3.2. Método FORM (First Order Reliability Method) 81

5.3.2.1. Transformação de Variáveis para o Espaço Reduzido 83

5.3.2.2. Busca do Ponto de Projeto 83

5.3.2.3. Fator de Importância das Variáveis 83

5.4. Geração da Superfície de Resposta 84

5.4.1. Superfície de Colocação Estocástica 86

5.5. Exemplos de Validação 88

5.5.1. Verificação da Importância das Variáveis $\quad 89$

5.5.2. Validação do Uso da Superfície de Resposta 90

5.5.3. Análise de Sensibilidade dos Parâmetros de Resistência 94

5.5.4. Análise de Confiabilidade Via Uso do Método de Elementos Finitos e Superfície de Resposta 96

6 Exemplos de Aplicação 100

6.1. Encosta de Coos Bay 100

6.2. Encosta da Vista Chinesa 110 
7 Conclusões e Sugestões 122

7.1. Conclusões 122

7.2. Sugestões para futuros trabalhos 124

8 Revisão Bibliográfica 125

A Apêndice 136

A.1 Fundamentos da Análise de confiabilidade 136

A.1.1 Variáveis Aleatórias 136

A.1.1.1 Características das Variáveis Aleatórias 136

A.1.1.2 Função Densidade de Probabilidade 138

$\begin{array}{ll}\text { A.1.1.3 Distribuições de Probabilidades } & 139\end{array}$ 


\section{Lista de figuras}

Figura 2.1- Relação tensão deformação para modelo rígido-plástico perfeito (Chen e Han, 1988). 22

Figura 2.2- Superfície de resistência no espaço das tensões principais. 23

Figura 2.3- Critério de escoamento de Mohr-Coulomb no plano $(\sigma, \tau)$.

Figura 2.4- Critério de resistência de Mohr-Coulomb com secção em forma de octógono. 25

Figura 2.5- Superfície de escoamento de Mohr-Coulomb: no espaço das tensões principais $(\mathrm{c}=0)$. 26

Figura 2.6- Superfície de plastificação de Drucker-Prager. 26

Figura 2.7- Envoltória tridimensional de resistência para solos não saturados (adaptado de Lu e Likos, 2004). 30

Figura 3.1- Área elementar de solo. $\quad 34$

Figura 3.2- Elemento de solo não saturado (adaptado Fredlund e Rahardjo, 1993). $\quad 36$

$\begin{array}{ll}\text { Figura 3.3- Curva Característica. } & 37\end{array}$

Figura 3.4- Histerese (adaptado, Reichardt e Timm, 2004). 38

Figura 3.5- Função da condutividade hidráulica. 39

Figura 3.6- Geometria e discretização para a validação de fluxo não saturado. 50

Figura 3.7- Curva caracteristica. $\quad 50$

Figura 3.8- Evolução da carga de pressão no tempo. 51

Figura 3.9- Geometria do exemplo de validação, malha de elementos

finitos e condições iniciais. $\quad 52$

Figura 3.10 - Evolução da carga de pressão no tempo para os nós 1, 2 e $3 . \quad 53$

Figura 4.1 Superfície de resistência e vetor de deformação plástica

(Carrion, 2009). 58

Figura 4.3 Cone quadrático. $\quad 64$

$\begin{array}{ll}\text { Figura 4.4 Elemento quadrilateral isoparamétrico. } & 67\end{array}$

Figura 4.5 - Geometria e malha de elementos finitos do talude infinito. $\quad 69$

Figura 4.6 - Estimativa do fator de segurança ao colapso mediante redução dos parâmetros de resistência. $\quad 71$

Figura 4.7 - Geometria do talude 2D. 72

Figura 4.8 - Malha de elementos finitos. 72

Figura 4.9 - Estimativa do fator de segurança ao colapso mediante redução 
dos parâmetros de resistência para o talude 2D. 73

Figura 5.1 - Função de falha. $\quad 76$

Figura 5.2 - Função densidade de probabilidade de g. 78

Figura 5.3 - Função distribuição acumulada da probabilidade de falha $g(X) . \quad 79$

Figura 5.4 - Pontos na simulação de Monte Carlo (Adaptado de Pereira, 2007).

Figura 5.5 - Representação gráfica do método FORM (a) espaço original e

(b) espaço normal padrão.

Figura 5.6- Interpolação com uso dos polinômios de Lagrange (adaptado de

Da Costa Pantoja, 2012).

Figura 5.7 - Gráfica da superfície de resposta. $\quad 88$

Figura 5.8- Modelo do talude infinito.

Figura 5.9- (a) Pontos da superfície de resposta, (b) superfície de $\begin{array}{ll}\text { resposta aproximada. } & 91\end{array}$

Figura 5.10- Índice de confiabilidade, talude infinito unidimensional. 92

Figura 5.11 - Diagrama de fluxo da metodologia aplicada para a determinação do índice de confiabilidade via superfície de resposta.

Figura 5.12 - (a) Pontos da superfície de resposta, (b) superfície de resposta aproximada; para as variáveis aleatórias $\mathrm{C}$ e $\phi$.

Figura 5.13- (a) Pontos da superfície de resposta, (b) superfície de resposta aproximada; para as variáveis aleatórias $\mathrm{C}$ e $\gamma$.

Figura 5.14 - (a) Pontos da superfície de resposta, (b) superfície de resposta aproximada; para as variáveis aleatórias $\phi$ e $\gamma$.

Figura 5.15- Índice de confiabilidade, para diversas combinações na geração da superfície de resposta de duas variáveis aleatórias. 96

Figura 5.16 - Talude infinito modelo bidimensional.

Figura 5.17- (a) Pontos da superfície de resposta, (b) superfície de resposta aproximada; do talude bidimensional na profundidade de $0,4 \mathrm{~m}$ da frente de umedecimento.

Figura 5.18- Índice de confiabilidade, talude infinito bidimensional. 99

Figura 6.1 - Geometria da encosta Coos Bay. 101

Figura 6.2 - Seção da malha de elementos finitos da encosta Coos Bay. 101

Figura 6.3 - Curvas característica e de condutividade hidráulica. 102

Figura 6.4 - Histograma da precipitação acontecida na encosta CB em 1996 (Adaptado de Borja et al., 2012).

Figura 6.5 - Variação da carga de pressão no tempo 24 horas do cenário 
de precipitações 1.

Figura 6.6 - Variação da carga de pressão no tempo 25.7 horas do cenário de precipitações 1.

Figura 6.7 - Variação da carga de pressão no tempo 13.6 horas do do cenário de precipitações 2 .

Figura 6.8 - Variação da carga de pressão no tempo 16.1 horas do cenário de precipitações 2.

Figura 6.9 - Variação da carga de pressão no tempo 2.5 horas do caso 3.106 Figura 6.10 - (a) Pontos da superfície de resposta, (b) superfície de resposta aproximada; para as variáveis aleatórias c' e $\phi$ '.

Figura 6.11 - Perfil geotécnico e geométrico da encosta da vista Chinesa.

Figura 6.12 - Seção da malha de elementos finitos da encosta da vista Chinesa.

Figura 6.14 - Curvas características (a) solo coluvionar e (b) solo residual (Adaptado de Velloso, 2007).

Figura 6.15 - Variação da carga de pressão no dia 3 de precipitação.

Figura 6.16 - Variação da carga de pressão no dia 5 de precipitação. $\quad 114$

Figura 6.17 - Variação da carga de pressão no dia 12 de precipitação. 115

Figura 6.18 - Variação da carga de pressão no dia 18 de precipitação. 115

Figura 6.19 - Variação da carga de pressão no dia 22 de precipitação.

116

Figura 6.20 - (a) Resultados do fator de segurança oa colapso (FS) da encosta da Vista Chinesa e (b) variação da carga de pressão no tempo.

Figura 6.21- (a) Pontos da superfície de resposta, (b) superfície de resposta aproximada; para as variáveis aleatórias c1' e c2'.

Figura 6.22 - (a) Resultados do $\beta$ da encosta da Vista Chinesa comparado com os (b) Fatores de segurança aso colapso (FS) via análise limite e (c) variação da carga de pressão no tempo. 


\section{Lista de tabelas}

Tabela 4.1 - Propriedades do material do talude infinito. 69

Tabela 4.2 - Redução de parâmetros de resistência do talude infinito. 70

Tabela 4.3 - Propriedades do material do exemplo. 72

Tabela 4.4 - Redução de parâmetros de resistência do talude infinito. 73

Tabela - 5.1 Relação do índice de confiabilidade e a probabilidade de falha

(USACE, 1999). 80

Tabela 5.2 - Variáveis utilizadas na análise de confiabilidade. 89

Tabela - 5.3 Fator de importância dos parâmetros. 90

Tabela 5.4 - Propriedades das variáveis aleatórias. 98

Tabela 6.1 - Resultados do Análise Limite para o caso 1.

Tabela 6.2 - Resultados do Análise Limite para o caso 2.

Tabela 6.3 - Resultados do Análise Limite para o caso 3.

Tabela 6.4 - Desvios padrões indicativos das propriedades do solo (Adaptado

$\begin{array}{ll}\text { de JCSS, 2006). } & 108\end{array}$

Tabela 6.5 - Propriedades das variáveis aleatórias da encosta Coos Bay. 108

Tabela-6.6 - Resultado da análise de confiabilidade do caso $1 . \quad 109$

Tabela-6.7 - Resultado da análise de confiabilidade do caso 2.

Tabela-6.8 - Resultado da analise de confiabilidade do caso 3.

Tabela 6.9 - Propriedades hidráulicas dos solos da vista Chinesa (Velloso,

2007). 113

Tabela 6.10 - Propriedades de resistência da encosta da Vista Chinesa. 116

Tabela 6.11 - Propriedades das variáveis aleatórias da encosta da Vista

Chinesa. 


\section{Lista de Símbolos}

\section{No critério de resistência}

$\begin{array}{lll}c & \text { Coesão } & {\left[\mathrm{MT}^{2} \mathrm{~L}^{-1}\right]} \\ c^{\prime} & \text { Coesão efetiva } & {\left[\mathrm{MT}^{2} \mathrm{~L}^{-1}\right]} \\ c^{*} & \text { Coesão aparente } & {\left[\mathrm{MT}^{2} \mathrm{~L}^{-1}\right]} \\ u_{a} & \text { Poro pressão de ar } & {\left[\mathrm{MT}^{2} \mathrm{~L}^{-1}\right]} \\ u_{w} & \text { Poro pressão da água } & {\left[\mathrm{MT}^{2} \mathrm{~L}^{-1}\right]} \\ F\left(\sigma_{i j}\right) & \text { Função de falha } & \\ \phi & \text { Ângulo de atrito } & \\ \phi^{\prime} & \text { Ângulo de atrito efetivo } & \\ \phi^{b} & \text { Parâmetro de resistência não saturada } & \\ \sigma & \text { Tensão normal } & {\left[\mathrm{MT}^{2} \mathrm{~L}^{-1}\right]} \\ \sigma_{1} \text { e } \sigma_{3} & \text { Tensões normais principais } & {\left[\mathrm{MT}^{2} \mathrm{~L}^{-1}\right]} \\ \sigma^{\prime} & \text { Tensão normal efetiva } & {\left[\mathrm{MT}^{2} \mathrm{~L}^{-1}\right]} \\ \tau & \text { Resistencia ao cisalhamento } & {\left[\mathrm{MT}^{2} \mathrm{~L}^{-1}\right]} \\ \chi & \text { Parâmetro de resistência não saturada } & {[-]}\end{array}$

\section{No fluxo saturado não saturado}

[B]

$C(h)$

$C_{S}$

$C_{w}$

$\{G\}$

$h$

$\hat{h}(x, z, t)$

$H_{t}$

[H]

$[J]$ $[K]$,

$[K(\theta)]$, $[K(h)], \quad K_{i 2}$

$k_{s}$

$n$

$n_{i}$

$n_{e}$

$N_{l}$

$\{q\}$

$\{Q\}$

$r, s$

Matriz que relaciona o gradiente

hidráulico com a

Capacidade de retenção especifica

Compressibilidade do solo

$\left[\mathrm{L}^{-1}\right]$

Compressibilidade do fluido

$\left[\mathrm{M}^{-1} \mathrm{~T}^{2 \mathrm{~L}}\right]$

Vetor associado aos gradientes de carga

de elevação nodais

Carga de pressão

$\left[\mathrm{M}^{-1} \mathrm{~T}^{2} \mathrm{~L}\right]$

$\left[\mathrm{L}^{3} \mathrm{~T}\right]$

Carga de pressão no interior do

elemento finito

Carga hidráulica total

Matriz de condutividade

Matriz Jacobiana dos elementos

$\left[\mathrm{L}^{2} \mathrm{~T}^{-1}\right]$

[L]

Tensor de condutividade hidráulica $\quad\left[\mathrm{LT}^{-1}\right]$

Condutividade hidráulica saturada $\quad\left[\mathrm{LT}^{-1}\right]$

Parâmetro empírico de Van Genuchten [-]

Componentes do vetor normal do

contorno $\Gamma_{\Gamma_{\mathrm{n}}}$

Número de nós do elemento

Funções de interpolação

Vetor de vazões específicas

Vetor de vazões nodais

$\left[\mathrm{L}^{2} \mathrm{~T}^{-1}\right]$

Coordenadas locais dos elementos

$\left[\mathrm{L}^{3} \mathrm{~T}^{-1}\right]$

[-] 


\begin{tabular}{|c|c|c|c|}
\hline & $R\left(h_{l}\right)$ & Resíduo do método de Galerkin & {$\left[\mathrm{L}^{3} \mathrm{~T}^{-1}\right]$} \\
\hline & $S$ & Grau de saturação & {$[-]$} \\
\hline & $S_{S}$ & $\begin{array}{l}\text { Coeficiente de armazenamento } \\
\text { específico }\end{array}$ & {$\left[\mathrm{L}^{-1}\right]$} \\
\hline & {$[S]$} & Matriz de massa & {$\left[\mathrm{L}^{2}\right]$} \\
\hline & $t$ & Tempo & {$[\mathrm{T}]$} \\
\hline & $u_{a}$ & Poro pressão de ar & {$\left[\mathrm{MT}^{2} \mathrm{~L}^{-1}\right]$} \\
\hline & $u_{w}$ & Poro pressão da água & {$\left[\mathrm{MT}^{2} \mathrm{~L}^{-1}\right]$} \\
\hline & $V$ & Volume total & {$\left[\mathrm{L}^{3}\right]$} \\
\hline & $V_{V}$ & Volume de vazios & {$\left[\mathrm{L}^{3}\right]$} \\
\hline & $V_{w}$ & Volume de agua & {$\left[\mathrm{L}^{3}\right]$} \\
\hline & $V_{s}$ & Volume de sólidos & {$\left[\mathrm{L}^{3}\right]$} \\
\hline & $W_{m}$ & $\begin{array}{l}\text { Pesos de ponderação na integração de } \\
\text { Gauss }\end{array}$ & {$[-]$} \\
\hline & $x_{i}$ & Coordenada global dos nós do elemento & [L] \\
\hline & $z_{i}$ & Coordenada global dos nós do elemento & {$[\mathrm{L}]$} \\
\hline & $z_{e}$ & Carga de elevação & \\
\hline & $\alpha$ & Parâmetro do modelo de Van Genuchten & {$\left[\mathrm{L}^{-1}\right]$} \\
\hline & $\xi$ & Porosidade do solo & {$[-]$} \\
\hline & $\rho_{w}$ & Massa especifica da água & {$\left[\mathrm{ML}^{-3}\right]$} \\
\hline 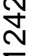 & $\delta_{i j}$ & Delta de Kroenecker & \\
\hline$\Phi$ & $\Delta t$ & Tamanho do passo do tempo & {$[\mathrm{T}]$} \\
\hline$\stackrel{\text { ol }}{\underline{z}}$ & $\Gamma$ & Contorn do modelo & \\
\hline 画 & $\Gamma_{D}$ & Contorno com condição de Dirichlet & \\
\hline $\bar{c}$ & $\Gamma_{N}$ & Contorno com condição de Neumman & \\
\hline 电 & $\theta$ & Umidade volumétrica & {$\left[\mathrm{L}^{3} \mathrm{~L}^{3}\right]$} \\
\hline ర్ల & $\theta_{s}$ & Umidade volumétrica saturada & {$\left[L^{3} L^{3}\right]$} \\
\hline 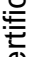 & $\theta_{s}$ & Umidade volumétrica saturada & {$\left[\mathrm{L}^{3} \mathrm{~L}^{3}\right]$} \\
\hline ¿ & $\theta_{r}$ & Umidade volumétrica residual & {$\left[\mathrm{L}^{3} \mathrm{~L}^{3}\right]$} \\
\hline$\stackrel{i}{\frac{1}{n}}$ & $\psi$ & Potencial total da água & {$\left[\mathrm{ML}^{2} \mathrm{~T}^{-2}\right]$} \\
\hline & $\psi_{g}$ & Potencial gravitacional & {$\left[\mathrm{ML}^{2} \mathrm{~T}^{-2}\right]$} \\
\hline & $\psi_{m}$ & Potencial matricial & {$\left[\mathrm{ML}^{2} \mathrm{~T}^{-2}\right]$} \\
\hline & $\psi_{o}$ & Potencial osmótico & \\
\hline & $\psi_{p}$ & Potencial de pressão & {$\left[\mathrm{ML}^{2} \mathrm{~T}^{-2}\right]$} \\
\hline & $\varpi$ & $\begin{array}{l}\text { Coeficiente que define o tipo de marcha } \\
\text { no tempo }\end{array}$ & \\
\hline & $\{\nabla H\}$ & Vetor gradiente da carga total & {$[\mathrm{L}]$} \\
\hline & $\Omega$ & Domínio do modelo & \\
\hline & $\Omega_{e}$ & Subdomínios & \\
\hline & $\mathrm{Na}$ an & ite no espaço cônico quadrático & \\
\hline & $c$ & Coesão & {$\left[\mathrm{MT}^{2} \mathrm{~L}^{-1}\right]$} \\
\hline & $c_{r}$ & Coesão reduzida & \\
\hline & {$[\mathcal{B}]$} & $\begin{array}{l}\text { Matriz de compatibilidade cinemática do } \\
\text { elemento }\end{array}$ & \\
\hline & {$[B]$} & Matriz de equilíbrio & \\
\hline & $\{b\}$ & Vetor dos carregamentos nodais & \\
\hline & {$[D]$} & Matriz de transformação para o espaço & \\
\hline
\end{tabular}


$\{d\}$

$\{f\}$

$F\left(\sigma_{i j}\right)$

[I]

$\left[N_{u}\right]$

$\left[N_{\sigma}\right]$

$\{p\}$

$r, s$

$\{\hat{\dot{u}}\}$

$\{\delta \dot{\varepsilon}\}$

$\{\delta \dot{d}\}$

$\dot{\varepsilon}_{i j}$

$\dot{\varepsilon}_{i j}^{e}$

$\dot{\varepsilon}_{i j}^{p}$

$\phi$

$\phi_{r}$

$\gamma$

$\lambda$

K

$\rho_{1}, \rho_{2}, \rho_{3}$

$\{\rho\}$

$\dot{\mu}$

$\omega$ cônico quadrático

Vetor da transformação para o espaço

cônico quadrático

Vetor das forças reais

Critério de resistência

Matriz de identidade

Matriz de interpolação das velocidades

Matriz de interpolação das tensões

Vetor da carga distribuída na superfície

Coordenadas paramétricas

Vetor de velocidade nodal

Vetor das velocidades de deformações

virtuais

Vetor dos deslocamentos virtuais

Velocidade de deformação total

Velocidade de deformação elástica

Velocidade de deformação plástica

Ângulo de atrito

Ângulo de atrito reduzido

Peso especifico

Fator de carga

Espaço cônico quadrático

Restrição no espaço cônico quadrático

Vetor da restrição do espaço cônico

quadrático

Fator de proporcionalidade (escalar)

Ângulo de inclinação

\section{Na análise de Confiabilidade}

$E(g(X)) \quad$ Valor esperado da função de falha

$g(X)$

Função de falha

$f(X)$

Função densidade de probabilidade (PDF)

$P(x) \quad$ Função distribuição acumulada CDF

$P_{f} \quad$ Probabilidade de falha

$R \quad$ Carga de resistência

$S \quad$ Carga solicitante

Superf $f_{(x, y)} \quad$ Superfície de resposta

$\operatorname{Var}(g(\boldsymbol{X})) \quad$ Variância da função de falha

$Y^{*} \quad$ Ponto de projeto

$\mathrm{z}$

Profundidade do frente de umedecimento

$\left\{X_{1}, X_{2}, \ldots, X_{n}\right\} \quad$ Vetor das variáveis aleatórias

$\beta$

$\delta_{x_{i}}$

$\mathcal{L}_{i}(x)$

Índice de confiabilidade

Coeficiente de variação

$\rho_{x_{i}, x_{k}}$

Polinômio de Lagrange

Coeficiente de correlação 


$\begin{array}{ll}\sigma_{g} & \text { Desvio padrão da função de falha } \\ \mu_{g} & \text { Valor médio da função de falha } \\ \Theta(x) & \text { Função aproximadora do polinômio de } \\ \omega & \text { Lagrange } \\ & \text { Ângulo de inclinação do talude }\end{array}$

